\title{
CARACTERIZACIÓN DE LA SOLUCIÓN DE UN PROBLEMA DE PROGRAMACIÓN LINEAL
}

\author{
Edinson Raúl, Montoro Alegre ${ }^{1}$, Willy David, Barahona Martínez ${ }^{2}$, \\ Luis Macha Collotupa ${ }^{3}$, Pedro Becerra Pérez ${ }^{4}$, Emilio Castillo Jiménez ${ }^{5}$, \\ Rocio De La Cruz Marcacuzco ${ }^{6}$, Gabriel Rodriguez Varillas ${ }^{7}$.
}

(Recibido: 15/10/2015 - Aceptado: 20/11/2015)

\begin{abstract}
Resumen: En los últimos años ha sido de gran interés el estudio de la complejidad de los algoritmos que resuelven los problemas de programación lineal como por ejemplo los algoritmos que siguen la trayectoria central y los algoritmos que han surgido como variantes del conocido algoritmo de Karmarkar. En este artículo presentamos una caracterización del punto solución de un problema de programación lineal a través de la longitud del tamaño de entrada del PPL.
\end{abstract}

Palabras Claves: Programación Lineal, Vértices de un conjunto Factible, Bases Determinantes.

\section{CHARACTERIZATION OF THE SOLUTION OF A LINEAR PROGRAMMING PROBLEM}

\begin{abstract}
In the last years it has been of great interest to study the complexity of algorithms that solve linear programming problems such as algorithms that follow the central path and algorithms that have emerged as variants known algorithm Karmarkar. In this article we present a characterization of point solution linear programming problem through the length of the input size of the PPL.
\end{abstract}

Key Words: Linear programming, vertices of a feasible set, Determinants Bases.

\section{Introducción}

Cuando se mide la complejidad de un algoritmo A que resuelve un problema algorítmico $\mathrm{P}$, el resultado siempre esta expresado en términos de la longitud del tamaño de la entrada del problema. El tamaño de la entrada del problema se refiere en términos generales a los valores de los parámetros que representan a una instancia del problema codificado por la máquina para su propio reconocimiento. Estos parámetros al ser codificados tienen una cierta longitud cada una, y al ser concatenadas todas a la vez conforman una cadena de cierta longitud. Esta longitud es el tamaño de la entrada del problema.

En los últimos años ha sido de gran interés el estudio de la complejidad de los algoritmos que resuelven los problemas de programación lineal como por ejemplo los algoritmos de Trayectoria Central y los algoritmos que surgen como variantes del algoritmo de Karmankar. En este artículo presentamos una caracterización del punto solución de un problema de programación lineal a través de la longitud del tamaño de entrada del Problema de Programación Lineal (PPL).

\footnotetext{
${ }^{1}$ UNMSM,Facultad de Ciencias Matemáticas, e-mail:edinsonmontoro@yahoo.com

${ }^{2}$ UNMSM, Facultad de Ciencias Matemáticas, e-mail:wilbara_73@yahoo.es

${ }^{3}$ UNMSM,Facultad de Ciencias Matemáticas, e-mail:Imachac@hotmail.com

${ }^{4}$ UNMSM, Facultad de Ciencias Matemáticas, e-mail:pbecerrap@unmsm.edu.pe

${ }^{5}$ UNMSM, Facultad de Ciencias Matemáticas, e-mail:ecastilloj@unmsm.edu.pe

${ }^{6}$ UNMSM, Facultad de Ciencias Matemáticas, e-mail:rodema_71@yahoo.es

${ }^{7}$ UNMSM, Facultad de Ciencias Matemáticas, e-mail:grodriguezv@unmsm.edu.pe
} 


\section{Preliminares}

\subsection{Tamaño de un Problema de Programación Lineal}

Consideramos el siguiente Problema de Programación Lineal

$$
(P P L):\left\{\begin{array}{c}
\min \quad C^{t} x \\
\text { s.a. } \quad A x=b \\
x \geq 0 .
\end{array}\right.
$$

donde

$$
A \in \mathbb{R}^{n \times m}, \quad b \in \mathbb{R}^{m} \quad \text { y } \quad C \in \mathbb{R}^{n} .
$$

El conjunto Factible del PPL es

$$
F=\left\{x \in \mathbb{R}^{n} / \quad A x=b ; \quad x \geq 0\right\}
$$

Sin pérdida de generalidad, podemos asumir que los coeficientes de la matriz $A$, del vector $b$ y del vector $C$ son enteros, pues cada PPL puede ser transformado en un problema equivalente con coeficientes enteros.

Definición 1: Para cada entero $n$ se define

$$
\operatorname{tamaño}(n)=1+\left\lceil\log _{2}(|n|+1)\right\rceil
$$

donde el primer 1 es colocado debido a que se necesita un bit para almacenar el signo de $n$. El tamaño $(n)$; representa el número de bits que se necesita para codificar el número $n$ en el sistema binario.

Definición 2: Dado un vector $u$ de orden $n \times 1$ y $B$ una matriz de orden $n \times k$ se define

$$
\begin{gathered}
\operatorname{tama\tilde {n}o}(u)=\sum_{i=1}^{n} \operatorname{tamaño} o\left(u_{i}\right) \\
\operatorname{tamaño}(B)=\sum_{i=1}^{n} \sum_{j=1}^{k} \operatorname{tamaño}\left(b_{i j}\right)
\end{gathered}
$$

Definición 3: (Tamaño de un PPL.) Se define como

$$
\operatorname{tamaño}(P P L)=\operatorname{tamaño}(A)+\operatorname{tamaño}(b)+\operatorname{tamaño}(C)
$$

Definición 4: Definimos la longitud $L$ como

$$
L=\operatorname{tama\tilde {n}o}\left(\operatorname{máx}_{A^{\prime}}\left\{\left|\operatorname{det}\left(A^{\prime}\right)\right|\right\}\right)+\operatorname{tama\tilde {n}o}\left(\operatorname{máx}_{1 \leq i \leq n}\left\{\left|b_{i}\right|\right\}\right)+\operatorname{tama\tilde {n}o}\left(\operatorname{máx}_{1 \leq j \leq n}\left\{\left|C_{j}\right|\right\}\right)+m+n .
$$

donde $A^{\prime}$ es cualquier submatriz cuadrada de $A$.

Teorema 1: Se cumplen las siguientes condiciones:

i) Si $n \in \mathbb{Z}$, entonces $|n| \leq 2^{\operatorname{tamaño}(n)-1}-1$.

ii) Si $u \in \mathbb{Z}^{n}$, entonces $\|u\| \leq\|u\|_{1} \leq 2^{\operatorname{tamaño}(u)-n}-1$.

iii) Si $A \in \mathbb{Z}^{n \times k}$, entonces $|\operatorname{det}(A)| \leq 2^{\operatorname{tamaño}(A)-n^{2}}-1$. 


\section{Demostración:}

Probemos (i): Por definición

$$
\begin{aligned}
& \operatorname{tamaño}(n)=1+\left\lceil\log _{2}(|n|+1)\right\rceil \\
& \operatorname{tamaño}(n)-1=\left\lceil\log _{2}(|n|+1)\right\rceil
\end{aligned}
$$

por otro lado si

$$
y=\left\lceil\log _{2}(|n|+1)\right\rceil
$$

entonces

$$
y \geq \log _{2}(|n|+1)
$$

luego pueden darse sólo dos casos:

1. Si $y=\log _{2}(|n|+1)$, entonces $2^{y}=|n|+1 \Rightarrow 2^{y}-1=|n|$

2. Si $y>\log _{2}(|n|+1)$, entonces $2^{y}=|n|+1 \Rightarrow 2^{y}-1>|n|$

De ambas se concluye $|n| \leq 2^{y}-1$, por lo tanto:

$$
|n| \leq 2^{\text {tamaño(n)-1 }}-1
$$

Probemos (ii): Tenemos que

$$
1+\|u\| \leq 1+\|u\|_{1}=1+\sum_{i=1}^{n}\left|u_{i}\right| \leq \prod_{i=1}^{n}\left(1+\left|u_{i}\right|\right)
$$

usando (i)

$$
1+\|u\| \leq \prod_{i=1}^{n} 2^{\sum_{i=1}^{n} \operatorname{tamaño}\left(u_{i}\right)-1}=2^{\operatorname{tamaño}(u)-n}
$$

Por lo tanto

$$
1+\|u\| \leq 2^{\text {tamaño(u)-n }} .
$$

Probemos (iii): Si $a_{1}, a_{2}, \ldots, a_{n}$ son las columnas de $A$ y como $|\operatorname{det}(A)|$ representa el volumen del poliedro generado por $a_{1}, a_{2}, \ldots, a_{n}$ entonces se tiene

$$
|\operatorname{det}(A)|=\prod_{i=1}^{n}\left\|a_{i}\right\|
$$

de donde usando (ii)

$$
\begin{gathered}
1+|\operatorname{det}(A)| \leq 1+\prod_{i=1}^{n}\left\|a_{i}\right\| \leq \prod_{i=1}^{n}\left(1+\left\|a_{i}\right\|\right) \\
1+|\operatorname{det}(A)| \leq \prod_{i=1}^{n} 2^{\operatorname{tamaño}\left(a_{i}\right)-n} \leq 2^{\sum_{i=1}^{n} \operatorname{tamaño}\left(a_{i}\right)-\sum_{i=1}^{n} n}=2^{\operatorname{tamaño}(A)-n^{2}}
\end{gathered}
$$

Por lo tanto

$$
|\operatorname{det}(A)| \leq 2^{\operatorname{tamaño}(A)-n^{2}}-1
$$


Teorema 2: Para cualquier $A \in \mathbb{R}^{m \times n}, \quad b \in \mathbb{R}^{m} \quad$ y $\quad C \in \mathbb{R}^{n}$ de un PPL se cumple

$$
L<\operatorname{tamaño}(P P L)
$$

Demostración: Si $B$ es submatriz cuadrada de $A$ entonces

$$
\operatorname{tamaño}(B) \leq \operatorname{tamaño}(A)
$$

Por el Lema anterior

$$
1+|\operatorname{det}(B)| \leq 2^{\operatorname{tamaño}(B)-n^{2}}
$$

como

$$
-n^{2} \leq-1 \quad \forall n \geq 1
$$

entonces

$$
1+|\operatorname{det}(B)| \leq 2^{\operatorname{tamaño}(B)-1}
$$

luego usando logaritmo tenemos

$$
\log (1+|\operatorname{det}(B)|) \leq \operatorname{tamaño}(B)-1<\operatorname{tamaño}(B) \leq \operatorname{tamaño}(A)
$$

por lo tanto

$$
\lceil\log (1+|\operatorname{det}(B)|)\rceil \leq \operatorname{tamaño}(A)
$$

Sea $v \in \mathbb{Z}^{p}$, entonces

$$
\begin{gathered}
\operatorname{tamaño}(v)=\sum_{i=1}^{p} \operatorname{tamaño}\left(v_{i}\right)=\sum_{i=1}^{p}\left(\log \left(\left|v_{i}\right|+1\right)+1\right)=\sum_{i=1}^{p}\left(\log \left(\left|v_{i}\right|+1\right)\right)+\sum_{i=1}^{p} 1 \\
\operatorname{tamaño}(v)=\sum_{i=1}^{p}\left(\log \left(\left|v_{i}\right|+1\right)\right)+p \geq\left\lceil\log \left(\left|v_{i}\right|+1\right)\right\rceil+p . \quad \forall i=1,2, \ldots, p .
\end{gathered}
$$

En particular para $j /\left|v_{j}\right|=\operatorname{máx}_{1 \leq i \leq p}\left\{\left|v_{i}\right|\right\}$ se obtiene

$$
\operatorname{tamaño}(v) \geq\left\lceil\log \left(\operatorname{máx}_{1 \leq i \leq p}\left\{\left|v_{i}\right|\right\}+1\right)\right\rceil+p
$$

luego, usando este hecho tenemos:

$\operatorname{tamaño}(P P L)=\operatorname{tamaño}(A)+\operatorname{tamaño}(b)+\operatorname{tamaño}(C) \geq$

$\left.\geq\lceil\log (1+|\operatorname{det}(B)|)\rceil+\left\lceil\log \left(\operatorname{máx}_{i}\left\{\left|b_{i}\right|\right\}+1\right)\right\rceil+m+\log \left(\operatorname{máx}_{i}\left\{\left|c_{i}\right|\right\}+1\right)\right\rceil+n=L$

Por lo tanto

$$
\operatorname{tamaño}(P P L) \geq L
$$

\section{Observación:}

Del teorema anterior se puede deducir:

i) $L \geq \log (1+|\operatorname{det}(B)|)+\log \left(\operatorname{máx}_{i}\left\{\left|b_{i}\right|\right\}+1\right)+\log \left(\operatorname{máx}_{i}\left\{\left|c_{i}\right|\right\}+1\right)+m+n$

$$
\begin{aligned}
& \geq \log (|\operatorname{det}(B)|)+\log \left(\operatorname{máx}_{i}^{i}\left\{\left|b_{i}\right|\right\}\right)+\log \left(\operatorname{máx}_{i}\left\{\left|c_{i}\right|\right\}\right)+\log _{2}\left(2^{m+n}\right) \\
& =\log \left(\left|\operatorname{det}\left(B^{*}\right)\right| \cdot\left|b_{i}^{*}\right| \cdot\left|c_{i}^{*}\right| \cdot\left(2^{m+n}\right)\right)
\end{aligned}
$$

Aplicando la exponencial concluimos que:

$$
2^{L}>\log \left(2^{m+n} \cdot\left|\operatorname{det}\left(B^{*}\right)\right| \cdot\left|b_{i}^{*}\right| \cdot\left|c_{i}^{*}\right|\right)
$$

ii) Para todo entero $n$ se cumple:

$$
2^{\operatorname{tamaño}(n)}>2^{\operatorname{tama} \tilde{n} o(n)-1}>2^{\operatorname{tamaño}(n-1)}-1 \geq|n|
$$

por lo tanto

$$
2^{\operatorname{tamaño}(n)}>|n|
$$




\subsection{Caracterización de la Solución de un PPL}

El siguiente teorema nos asegura que los vértices de la región factible de un problema de programación lineal en forma estandard tienen una representación compacta.

Teorema 3: Sea $x$ un vértice del polítopo definido por $A x=b, x>0$, es de la forma

$$
x^{t}=\left(\frac{p_{1}}{q} ; \frac{p_{2}}{q} ; \ldots ; \frac{p_{n}}{q}\right)
$$

donde:

$$
q, p_{i} \in \mathbb{N} \quad \text { y } \quad 0 \leq p_{i} \leq 2^{L} ; \quad 1 \leq q<2^{L} ; \quad \forall i=1,2, \ldots, n .
$$

Demostración: Por ser $x$ un vértice, entonces tiene que ser una solución básica factible(teorema de PPL). Por lo tanto existe una matriz base $B$ asociada a dicho vértice talque

$$
x=\left(\begin{array}{lll}
x_{B} & \vdots & 0
\end{array}\right)=\left(\begin{array}{lll}
B^{-1} b & \vdots & 0
\end{array}\right)
$$

De aquí deducimos que

$$
p_{j}=0, \quad \forall j \in I_{N} .
$$

Sólo nos falta ver como son los $p_{j}$ para los índices básicos $j \in I_{B}$.

Por el álgebra lineal sabemos que

$$
x_{B}=B^{-1} b=\frac{\operatorname{Cof}(B) \cdot b}{\operatorname{det}(B)}
$$

donde $\operatorname{Cof}(B)$ es la matriz de cofactores de $B$ y cada entrada de la matriz cofactor consiste de un determinante de alguna submatriz de $A$.

Sea $q=|\operatorname{det}(B)|$, entonces $q$ es un entero, puesto que las entradas de $B$ son enteros así $q \geq 1$.

Por otro lado como $B$ es invertible y debido a (ii) de la observación se cumple

$$
|q|=|\operatorname{det}(B)| \leq\left|\operatorname{det}\left(B^{*}\right)\right|<2^{\operatorname{tamaño}\left(\left|\operatorname{det}\left(B^{*}\right)\right|\right)}<2^{L}
$$

Luego, el vector $P_{B}=\left(p_{1}, p_{2}, \ldots, p_{j}\right), j \in I_{B}$ es

$$
P_{B}=q x_{B}=\operatorname{Cof}(B) \cdot b
$$

De aquí

$$
\begin{aligned}
\left|p_{i}\right| & \leq \sum_{j=1}^{m}\left|\operatorname{Cof}(B)_{i j}\right|\left|b_{j}\right| \\
& \leq \sum_{j=1}^{m}\left(\operatorname{máx}_{j}\left\{\left|\operatorname{Cof}(B)_{i j}\right|\right\}\right)\left(\operatorname{máx}_{j}\left\{\left|b_{j}\right|\right\}\right) \\
& =\sum_{j=1}^{m}\left(\left|\operatorname{det}\left(B^{*}\right)\right|\right)\left(\left|b_{k}^{*}\right|\right) \\
& =\left(\left|\operatorname{det}\left(B^{*}\right)\right|\left|b_{k}^{*}\right|\right) \sum_{j=1}^{m} 1 \\
& =\left(\left|\operatorname{det}\left(B^{*}\right)\right|\left|b_{k}^{*}\right|\right) m \\
& <\left|\operatorname{det}\left(B^{*}\right)\right|\left|b_{k}^{*}\right| 2^{\text {tamaño }(m)}<2^{L}
\end{aligned}
$$

Por lo tanto:

$$
\left|p_{i}\right|<2^{L} \quad \forall i \in I_{B}
$$


Teorema 4: Sean $x_{1}$ y $x_{2}$ los vértices de la región factible, es decir $A x=b, x_{1}, x_{2}>0$. Si $C^{t} x_{1} \neq C^{t} x_{2}$, entonces:

$$
\left|C^{t} x_{1}-C^{t} x_{2}\right|>2^{-2 L}
$$

Demostración: $x_{1}$ y $x_{2}$ por ser vértices entonces son soluciones básicas,

$$
\begin{aligned}
& x_{1}=\left(\frac{p_{1}^{\prime}}{q_{1}}, \frac{p_{2}^{\prime}}{q_{1}}, \ldots, \frac{p_{k}^{\prime}}{q_{1}}, 0,0,0, \ldots, 0\right) \\
& x_{2}=\left(\frac{p_{1}^{\prime \prime}}{q_{2}}, \frac{p_{2}^{\prime \prime}}{q_{2}}, \ldots, \frac{p_{k}^{\prime \prime}}{q_{2}}, 0,0,0, \ldots, 0\right)
\end{aligned}
$$

con $q_{1} \geq 1, \quad q_{2} \geq 1$ y $q_{1}<2^{L}, \quad q_{2}<2^{L}$ (debido al Teorema 3 ).

Luego $q_{1} \cdot q_{2} \geq 1$ así $\frac{1}{q_{1} q_{2}}>\frac{1}{2^{L}, 2^{L}}$ entonces

$$
\begin{gathered}
\left|C^{t} x_{1}-C^{t} x_{2}\right|=\left|q_{1} \frac{C^{t} x_{1}}{q_{1}}-q_{2} \frac{C^{t} x_{2}}{q_{2}}\right|=\left|\frac{q_{1} q_{2}\left(C x_{1}-C x_{2}\right)}{q_{1} q_{2}}\right| \\
\geq \frac{\left|C x_{1}-C x_{2}\right|}{q_{1} q_{2}} \geq \frac{1}{q_{1} q_{2}}>\frac{1}{2^{L}, 2^{L}}=2^{-2 L} \\
\left|C^{t} x_{1}-C^{t} x_{2}\right|>2^{-2 L} .
\end{gathered}
$$

\section{Resultado Principal}

A continuación damos el teorema principal:

Teorema 5: Sea

$$
Z=\min \left\{C^{t} x / A x=b ; x \geq 0\right\}
$$

y supongamos que $x$ es un vector factible, es decir

$$
A x=b \text { y } x \geq 0 \text { tal que } C^{t} x \leq Z+2^{-2 L}
$$

entonces se cumple:

Si $x^{*}$ es un vector factible tal que $C^{t} x^{*} \leq C^{t} x$, entonces $x^{*}$ es una solución óptima del PPL.

Demostración: Probemos por contradicción:

Supongamos que $x^{*}$ no es el vértice óptimo, y supongamos que $\widetilde{x}$ es el vértice óptimo, entonces

$$
C^{t} \widetilde{x}=z .
$$

Como

$x^{*}$ no es el óptimo, entonces

$$
C^{t} x^{*} \neq C^{t} \widetilde{x}
$$

y por el teorema 4 se cumple

$$
C^{t} x^{*}-C^{t} \widetilde{x}>2^{-2 L}
$$

Luego

$$
C^{t} x^{*}>C^{t} \widetilde{x}+2^{-2 L}
$$

por hipótesis

$$
C^{t} x^{*}>z+2^{-2 L} \geq C^{t} x
$$


entonces

$$
C^{t} x^{*}>C^{t} x
$$

lo cual es una contradicción.

Por lo tanto $x^{*}$ es el vértice óptimo.

Del Teorema anterior podemos deducir lo siguiente: Si $x^{*}$ es el vértice óptimo y $x$ es un vértice tal que

$$
C^{t} x \leq C^{t} x^{*}+2^{-2 L}
$$

entonces:

$$
x \text { es el óptimo. }
$$

Lo que implica

$$
\left|C^{t} x-C^{t} x^{*}\right| \leq 2^{-2 L}
$$

o equivalentemente

$$
\left|C^{t} x-z\right| \leq 2^{-2 L}
$$

Entonces, la última desigualdad nos dice que si hallamos un vector factible que produce un valor en la función objetivo que posee un valor absoluto menor o igual a $2^{-2 L}$ con respecto al valor óptimo, entonces dicho vector es el óptimo.

\section{Conclusiones}

Concluimos que existe una caracterización del punto óptimo de un problema de programación lineal en función del tamaño de entrada del problema.

Este artículo será de gran interés y motivación para seguir nuevos linderos en esta área de investigación. 


\section{REFERENCIAS BIBLIOGRÁFICAS}

[1] M. Bazarda, H. Sherali, AND C. Shetty.; Nonlinear Programming, Theory and Aplications, John Wiley \& Sons, New York, second ed. 1993.

[2] D. P. Bertsekas., Nonlinear Programming, Athena Scientific, Belmont Mass, 1995.

[3] R. Bulirsch And J. Stoer., Introduction to Numerical Analysis; Springer- Verlag. New York, 1980.

[4] A. Forsgren AND P.E. Gill., Primal-dual interior Methods for nonconvex nonlinear Programming, SIAM , Journal on Optimization,8,(1998) pp.1132-1152.

[5] R. T. Rockafellar., The multiplicar method of Hertenes and Poud Applied to Convex Programming Journal of Optimization theory and Applications,12,(1973) pp.555-562.

[6] Van Tiel J.; Convex Analysis, John Wiley and Sons. Northem Ireland at the Universities Press, 1984. 